\section{Crianças em Unidade de Terapia Intensiva de um Hospital Público da cidade de São Paulo: aspectos epidemiológicos durante internação: uma análise de 329 neonatos}

\section{Children in an Intensive Care Unit of a Public Hospital in the city of São Paulo: epidemiological aspects during hospitalization: an analysis of 329 neonates}

\author{
Cintia Portela Disessa, Ana Paula Ribeiro, Jane de Eston Armond, \\ Márcia Mathias Castro, Neil Ferreira Novo, Yara Juliano
}

\section{RESUMO:}

Objetivo: Introdução: A Unidade de Terapia Intensiva Pediátrica de hospitais públicos pertencente ao Sistema Único de Saúde, geralmente, atende crianças com as mais variadas patologias, sendo referência para muitos serviços da região metropolitana de São Paulo, mas pouco se conhece sobre as características epidemiológicas para assistência da criança dentre as várias especialidades clínicas durante período de internação.Objetivos: Investigar crianças na unidade de terapia intensiva de um hospital público em seus aspectos epidemiológicos durante hospitalização direcionados para os diagnósticos envolvidos, taxa de óbito e os fatores de risco para mortalidade. Método: Estudo transversal, descritivo e quantitativo. A população de estudo foi constituída de 329 crianças internadas em uma unidade de terapia intensiva pediátrica (UTIP) no período de dois anos consecutivos (2016 e 2017). As variáveis analisadas foram: gênero, faixa etária, diagnóstico, tempo de internação, reinternação, procedência, sazonalidade dos agravos, necessidade de atendimento multidisciplinar, uso de ventilação mecânica, escore de risco de morte (PIM2) e evolução. Na análise estatística foram utilizados os testes do Quiquadrado e de Mann-Whitney e considerou-se o nível de significância $p<0,05$. Resultados: Das 329 internações, $56,53 \%$ eram do gênero masculino e 41,34\% lactentes, ambos predominaram as hospitalizações. Os distúrbios respiratórios foram os mais prevalentes, caracterizando $52,28 \%$ das internações. Verificou-se que a porcentagem de doenças neurológicas e queimaduras foram significantemente maiores do que as patologias respiratórias e o grupo "outras" em 2016 ( $p=0,008$ ). Em 2017 as porcentagens de doenças neurológicas foram significantemente maiores do que as doenças respiratórias e queimaduras $(p=0,002)$. A porcentagem de óbito das doenças neurológicas $(32,3 \%)$ foi significantemente maior em relação aos outros grupos de patologias $(p=0,003)$. Conclusões: Houve predominância de crianças internadas na faixa etária dos menores de um ano, do gênero masculino e com doenças respiratórias, embora a mortalidade foi superior nas patologias neurológicas. 0 escore de risco de morte PIM 2 mostrou-se um bom preditor de mortalidade em UTI pediátrica.

PALAVRAS-CHAVE: Epidemiologia; Criança; Fatores de Risco; Unidade de Terapia Intensiva.

\section{ABSTRACT}

Objective: Introduction: The Pediatric Intensive Care Unit of public hospitals belonging to the Unified Health System usually serves children with various pathologies, being a reference for many services in the metropolitan region of São Paulo, but little is known about the epidemiological characteristics for care. Objectives: To investigate children in the intensive care unit of a public hospital on their epidemiological aspects during hospitalization, focusing on the diagnoses involved, the death rate and the risk factors for mortality. Method: Cross-sectional, descriptive and quantitative study. The study population consisted of 329 children admitted to a pediatric intensive care unit (PICU) over two consecutive years (2016 and 2017). The variables analyzed were: gender, age group, diagnosis, length of stay, readmission, origin, seasonality of the diseases, need for multidisciplinary care, use of mechanical ventilation, risk of death score (PIM2) and evolution. Statistical analysis was performed using the chi-square and Mann-Whitney tests and the significance level $p<0.05$ was considered. Results: Of 329 hospitalizations, $56.53 \%$ were male and $41.34 \%$ infants, both hospitalizations predominated. Respiratory disorders were the most prevalent characterizing $52.28 \%$ of hospitalizations. It was found that the percentage of neurological diseases and burns were significantly higher than respiratory pathologies and the "other" group in $2016(p=0.008)$. In 2017, the percentages of neurological diseases were significantly higher than respiratory diseases and burns $(p=0.002)$. The percentage of death from neurological diseases $(32.3 \%)$ was significantly higher compared to other pathological groups $(p=0.003)$. Conclusions: There was a predominance of children hospitalized in the age group of under one year old, male and with respiratory diseases although mortality was higher in neurological pathologies. The PIM 2 risk of death score proved to be a good predictor of mortality in a PICU.
Conflito de Interesse: Não há conflito de interesse 


\section{INTRODUÇÃO}

A Unidade de Terapia Intensiva (UTI) surgiu com a necessidade de oferecer cuidados especiais a pacientes criticamente doentes, o que implica em uma equipe altamente treinada, equipamentos específicos e instalações projetadas para tal finalidade. Nesse ambiente hospitalar, preconiza-se a redução nos índices de mortalidade das crianças por doenças específicas, bem como o período de permanência na UTI¹.

Sabe-se que em hospitais públicos pertencente ao SUS (Sistema Único de Saúde), a Unidade de Terapia Intensiva Pediátrica - UTIP, atende crianças com as mais variadas patologias, sendo referência para muitos serviços da região metropolitana de São Paulo, em virtude de suas características e referências para o atendimento clinico especializado. Muitos hospitais públicos com UTIP têm como assistência diferenciada ações multidisciplinares constituída por médicos, enfermeiros, técnicos ou auxiliares de enfermagem, fisioterapêutas, terapêuta ocupacional, fonoaudióloga, nutricionista, psicóloga e assistente social², mas pouco se conhece e compreende acerca dos aspectos epidemiológicas das crianças internadas na UTIP nos seus diferentes diagnósticos.

O cuidado intensivo multidisciplinar proporciona uma modalidade assistencial que segue diretrizes e normas técnicas para garantir a segurança e a qualidade dos serviços prestados aos diagnósticos clínicos em unidades de alta complexidade, como é o caso da UTIP². Na garantia dos serviços prestados durante período de hospitalização, um estudo realizado no estado de Pernambuco verificou que as principais causas de internação de crianças na UTIP foram: neoplasias, malformações congênitas, doenças infectoparasitárias, do aparelho respiratório e do aparelho digestivo³. Neste contexto, observa-se que características populacionais e ambientais se diferem nas diferentes regiões, e, esse fato deve ser ponderado para melhor compreensão das causas de internações de crianças na UTIP. Estudos nacionais nesse âmbito, porém de magnitude regional, ressaltaram a prevalência de doenças na infância, sendo elas: infecção respiratória aguda (IRA), anemia, desidratação, diarréia e desnutrição²-5.

De acordo com Molina et al. (2019)² é importante compreender o processo saúde-doença na assistência terciária de forma que gere impacto para sociedade, fornecendo indicadores que sirvam de suporte ao planejamento, administração e avaliação das ações de saúde preventivas. Neste racional, compreender o coeficiente de mortalidade infantil é um dos maiores indicadores de saúde utilizados universalmente para medir o nível de saúde de uma população $0^{5,6}$. A tendência de redução da mortalidade infantil no Brasil iniciada na década de oitenta mantém-se nas décadas seguintes, passando de 48 óbitos por mil nascidos vivos em 1990 para 29,6 em 2000 e menor que 15 em 2015․ Analisando-se a taxa de mortalidade infantil no Município de São Paulo (MSP) observa-se que esse coeficiente foi de 11,3 óbitos por mil nascidos vivos em 2017, de 10,8 em 2018 e de 11,0 entre 2019 e 2020. Os maiores coeficientes foram encontrados nas regiões Leste, Norte e Sul do estado de São Paulo, fato este, que reforça a importância de identificar os seus determinantes ${ }^{8}$. 
A carência de literatura científica contendo dados epidemiológicos em UTIP, ainda permanece em evidência ${ }^{5}$. Assim, pesquisas devem impulsionar novas frentes de estudo na área para melhor acompanhamento e delineamento da população envolvida, guiando condutas e reflexões dos profissionais no contexto prático e científico. Frente a esse panorama, este estudo tem por objetivo investigar [o perfil de crianças] hospitalizadas na terapia intensiva de um hospital público em seus aspectos epidemiológicos direcionados para os diagnósticos envolvidos, taxa de óbito e os fatores de risco para mortalidade. A hipótese do estudo foi que as crianças hospitalizadas na UTIP apresentariam maior predominância das doenças respiratórias com redução da taxa de óbito durante o período de internação.

\section{MÉTODO}

\section{Design e Recrutamento da Amostra}

Trata-se de um estudo transversal e de dados quantitativos com características descritiva e analítica. A população de estudo foi constituída de crianças internadas na unidade de terapia intensiva pediátrica (UTIP) do Hospital Municipal Doutor Carmino Caricchio (HMCC) no período de dois anos (janeiro de 2016 a dezembro de 2017). Foram incluídos na amostra 329 internações conforme os critérios de inclusão a seguir: a) crianças hospitalizadas na UTIP com idade igual ou maior que um mês até 14 anos 11 meses e 29 dias; b) crianças com mais de uma admissão na UTIP; c) pacientes que foram internados anteriormente ao início da pesquisa e mantiveram-se internados após o seu início. Foram excluídos da amostra: a) pacientes hospitalizados com idade menor que um mês de vida; b) crianças que foram institucionalizadas para cuidados pós- realização de exames específicos; c) pacientes que tiveram tempo de permanência inferior a 12 horas.

Os dados foram coletados a partir do software Monitor Epimed® e do Livro de Registro de Internações da Unidade de Terapia Intensiva Pediátrica. Os resultados obtidos foram transcritos no software Microsoft Excel® (versão 16.0). As variáveis analisadas foram: gênero, faixa etária, diagnóstico, tempo de internação, reinternação, procedência, sazonalidade dos agravos, necessidade de atendimento multidisciplinar, uso de ventilação mecânica, escore de risco de morte e evolução.

Para a análise descritiva dos dados as variáveis categóricas foram expressas como frequências absolutas e percentuais, média e mediana, já para a análise estatística não paramétrica realizou-se a aplicação do teste da Partição do Qui-quadrado e teste de Mann- Whitney, utilizando o IBM SPSS Statistics (versão 26), considerando um nível de significância $p<0,059,10$.

Este estudo foi submetido e aprovado pelo Comitê de Ética em Pesquisa (CEP) da Universidade Santo Amaro e do Hospital Municipal Dr. Carmino Caricchio/Secretaria Municipal de Saúde de São Paulo sob o CAAE:94743418.0.0000.0081. 
O Termo de Consentimento Livre e Esclarecido foi dispensado por se tratar de dados secundários, sem intervenção ou contato direto com os pacientes, com risco mínimo aos mesmos. Desta forma, a pesquisadora principal assinou um Termo de Sigilo e Confiabilidade para o uso das informações coletadas.

\section{RESULTADOS}

O número inicial de crianças foi de 388 crianças, mas devido os critérios de exclusão, o número total de participantes incluídos foi de 329 crianças, sendo 163 no ano de 2016 e 166 em 2017, dos quais, 186 eram do gênero masculino, correspondendo a $56,53 \%$ da amostra, e 143 do gênero feminino, cerca de $43,47 \%$ do total. Observou-se que as crianças com idade entre 1 mês completo até 11 meses e 29 dias, predominam entre as internações na UTIP em questão sendo responsáveis por 41,34\% das mesmas, seguidos das crianças com faixa etária entre 1 - 3 anos que correspondem a $27,66 \%$, os pueris com idade maior ou igual a 8 anos foram $17,93 \%$ da amostra e o intervalo etário entre 4 a 7 anos tem a menor freqüência de internações alcançando 13,07\% destas conforme Tabela 1.

Tabela 1: Crianças internadas na UTI segundo gênero e faixa etária nos anos 2016 e 2017

\begin{tabular}{lcc}
\hline VARIÁVEIS & NÚMERO $(\mathbf{N})$ & PORCENTAGEM $(\%)$ \\
\hline Gênero & 186 & \\
Masculino & 143 & 56,53 \\
Feminino & & 43,47 \\
\hline Faixa etária & \\
$<1$ ano & 136 & 41,34 \\
$1-3$ anos & 91 & 27,66 \\
$4-7$ anos & 43 & 13,07 \\
$\geq 8$ anos & 59 & 17,93 \\
\hline TOTAL & 329 & 100,00 \\
\hline
\end{tabular}

Na Tabela 2 verifica-se que a porcentagem de óbitos por doenças neurológicas e queimaduras que não diferem entre si, foram significativamente maiores do que as doenças respiratórias e o grupo "outras" em 2016. As doenças respiratórias e neurológicas observadas nas crianças avaliadas, não tinham histórico no prontuário de parto de alto risco, mas 10\% apresentavam histórico de prematuridade.

No entanto, as porcentagens de doenças neurológicas foram significativamente maiores do que as doenças respiratórias e queimaduras no ano de 2017. Nos dados de 2016 observa-se as seguintes partições:

$1^{a}$ partição: (Doenças Neurológicas+Queimaduras) > (Doenças Respiratórias+Outras) $X^{2}=11,34(p=0,008)$

$2^{a}$ partição: (Doenças Respiratórias $X$ Outras) $X^{2}=0,23 p=0,631$. 
Já na partição do quiquadrado dos dados referentes à 2017 temos:

1a partição: (Doenças Neurológicas > Doenças Respiratórias + Queimaduras + Outras) $X^{2}=13,66(p=0,002)$

$2^{\mathrm{a}}$ partição: (Doenças Respiratórias X Queimaduras) $X^{2}=1,46 p=0,226$.

Não houve diferença significativa entre 2016 e 2017 em relação aos óbitos de cada grupo de doença.

Tabela 2: Crianças internadas na UTI segundo óbito ou alta e a doença referida nos anos 2016 e 2017. Resultado do teste do Quiquadrado $\left(x^{2}\right)$ e do teste exato de Fisher $(p)$.

\begin{tabular}{ccccc|ccccc}
\hline \multirow{2}{*}{ Doença } & \multicolumn{5}{c|}{2016} & \multicolumn{3}{c}{2017} \\
Óbito & Alta & Total & \%óbito & Óbito & Alta & Total & \%óbito & \\
\hline Respiratória & 4 & 81 & 85 & 4,7 & 10 & 77 & 87 & 11,5 & $\mathrm{p}=0,174$ \\
Neurológica & 5 & 14 & 19 & 26,3 & 5 & 7 & 12 & 41,7 & $\mathrm{p}=0,447$ \\
Queimadura & 3 & 11 & 14 & 21,4 & 2 & 19 & 21 & 9,5 & $\mathrm{p}=0,369$ \\
Outras & 3 & 42 & 45 & 6,7 & 2 & 4 & 46 & 4,3 & $\mathrm{p}=0,676$ \\
Total & 15 & 148 & 163 & 9,2 & 19 & 147 & 166 & 11,4 & \\
\hline \multicolumn{7}{c}{$\mathrm{X}^{2}=11,57(\mathrm{p}=0,009)$} & $\mathrm{X}^{2}=13,17(\mathrm{p}=0,040)$ &
\end{tabular}

O tempo de internação variou de 1 a 1470 dias durante o período dos anos 2016 e 2017, com média de 17,2 dias e mediana de 7 dias. A tabela 3 apresenta a média e mediana do tempo de internação dos pacientes da amostra.

Tabela 3: Crianças internadas na UTI segundo tempo de permanência nos anos 2016 e 2017.

\begin{tabular}{ccc}
\hline Ano & $\begin{array}{c}\text { Tempo de internação (em dias) } \\
\text { média }\end{array}$ & $\begin{array}{c}\text { Tempo de internação (em dias) } \\
\text { mediana }\end{array}$ \\
\hline 2016 & 22,9 & 6 \\
2017 & 11,8 & 7 \\
Biênio $(2016+2017)$ & 17,2 & 7 \\
\hline
\end{tabular}

As porcentagens de óbito observadas em crianças procedentes do Pronto Socorro Infantil e de pacientes provenientes de serviços externos foram significativamente maiores do que os observados em pacientes procedentes da enfermaria pediátrica e de outros setores hospitalares (Tabela 4). 
Tabela 4: Crianças internadas na UTI segundo óbito ou alta e a procedência nos biênio 2016 e 2017.

\begin{tabular}{|c|c|c|c|c|}
\hline \multirow{2}{*}{ Procedência } & \multicolumn{2}{|c|}{ Óbito } & \multirow{2}{*}{ Total } & \multirow{2}{*}{$\%$ Óbitc } \\
\hline & Sim & Não & & \\
\hline Pronto Socorro Infantil & 16 & 109 & 125 & 12,8 \\
\hline Enfermaria Pediátrica & 2 & 67 & 69 & 2,9 \\
\hline${ }^{*}$ Outros setores hospitalares & 3 & 55 & 58 & 5,2 \\
\hline Externo & 13 & 64 & 77 & 16,9 \\
\hline Total & 34 & 295 & 329 & \\
\hline
\end{tabular}

Outros setores hospitalares correspondem a setores internos da própria instituição como: centro cirúrgico, centro de tratamento de queimados e unidade de terapia intensiva neonatal.

O uso da ventilação mecânica (VM) no grupo de óbitos (100\%), foi significativamente maior do que o observado nos demais grupos de evolução. No quesito uso de ventilação mecânica verificou-se que das 329 crianças internadas, $138(41,94 \%)$ tiveram uso do suporte ventilatório invasivo, enquanto $191(58,06 \%)$ não utilizaram essa modalidade terapêutica (Tabela 5).

Dos pacientes submetidos à ventilação mecânica 10,33\% tiveram o óbito como desfecho, 1,82\% foram transferidos para outro serviço, 6,69\% tiveram alta direto para o domicílio e $23,10 \%$ evoluíram com alta para outro setor do HMCC conforme apresentado na tabela 5. Foi observado que todas as crianças que foram à óbito no período estudado foram submetidas à ventilação mecânica como medida de suporte avançado de vida. Já a maioria dos pacientes expostos à ventilação mecânica durante a internação apresentou como evolução a alta interna hospitalar, ou seja, foram transferidos para setores do próprio hospital (Tabela 5).

Tabela 5: Evolução das crianças internadas na UTI segundo uso de ventilação mecânica nos anos 2016 e 2017.

\begin{tabular}{|c|c|c|c|c|}
\hline \multirow[t]{2}{*}{ Evolução } & \multicolumn{2}{|c|}{$\begin{array}{c}\text { Ventilação } \\
\text { Mecânica (VM) }\end{array}$} & \multirow[t]{2}{*}{ Total } & \multirow[t]{2}{*}{$\%$ uso de VM } \\
\hline & Sim & Não & & \\
\hline Alta domiciliary & 22 & 29 & 51 & 43,1 \\
\hline Alta interna hospitalar & 76 & 155 & 231 & 32,9 \\
\hline Transferência externa & 6 & 7 & 13 & 46,2 \\
\hline Óbito & 34 & 0 & 34 & 100,0 \\
\hline Total & 138 & 191 & 329 & 41,9 \\
\hline
\end{tabular}

O valor médio do PIM 2 entre as crianças que foram à óbito $(30,1)$ foi significantemente maior do que as que receberam alta $(3,3)$. Os valores da média e mediana do PIM 2 entre as crianças que foram à óbito foi significativamente maior do que as que receberam alta.

A análise do escore de risco de morte (PIM 2) foi realizada em conjunto para os anos 2016 e 2017 por não haver diferença significativa para os diagnósticos nesse período. O Teste de Mann-Whitney foi usado para comparar os valores 
do PIM 2 para as crianças que foram à óbito e aquelas que tiveram alta, sendo evidenciado pelo resultado encontrado que se trata de um bom indicador preditivo de mortalidade em UTIP, com $Z=6,85$ ( $p=0,0000)$. Dentre os pacientes que foram à óbito, o valor máximo encontrado do escore PIM 2 na amostra foi 98,43 e o valor mínimo 0,42. Entre as crianças que foram de alta o valor máximo do escore foi 64,67 e o mínimo 0,13, tabela 6.

Tabela 6: Crianças internadas na UTI segundo óbito ou alta e os valores da média e mediana, máximo e mínimo do escore de mortalidade (PIM 2) nos anos 2016 e 2017.

\begin{tabular}{lll}
\hline & Óbito & Alta \\
\hline Média & 30,1 & 3,3 \\
Mediana & 11,5 & 1,3 \\
Máximo & 98,4 & 64,6 \\
Mínimo & 0,42 & 0,13 \\
\hline \multicolumn{3}{c}{ Teste de Mann - Whitney (óbito $x$ alta) } \\
\hline
\end{tabular}

\section{DISCUSSÃO}

Este estudo descreveu o perfil epidemiológico de crianças internadas na UTI Pediátrica de um Hospital Público do município de São Paulo, entre o período de 2016 e 2017. Retratou as características da amostra quanto ao gênero, faixa etária, diagnóstico, tempo de internação, reinternação, procedência, atendimento multidisciplinar, sazonalidade, uso de ventilação mecânica, escore de risco de morte e sua relação com a mortalidade. Os dados gerais são similares aos encontrados em estudos realizado por Veloso et al. (2019)11 em crianças na UTIP, no qual os autores ressaltaram que a malformação congênita, a gestação múltipla e o sexo masculino do recém-nascido mostraram-se determinantes à mortalidade neonatal no Brasil, entretanto não constituem fatores modificáveis.

Quanto aos diagnósticos que motivaram as internações na UTIP, observadas neste estudo, os mesmos estão de acordo com os descritos por Batista et al. (2015) ${ }^{12}$, de Molina et al. (2008)2 e de Sena et al. (2006) ${ }^{13}$, cuja a prevalência foi maior no grupo de doenças respiratórias presentes neonatos prematuros e em crianças com idades acima de 28 dias. Os aspectos biológicos que tornam essa população mais vulnerável é o baixo peso ao nascer, fator determinante na ocorrência da mortalidade neonatal no Brasil, assim como outros fatores modificáveis, a exemplo da asfixia perinatal, prematuridade, intercorrências durante a gestação, pré-natal feito de forma inadequada, parto cesariano e idade materna avançada ${ }^{2,13,14}$. No presente estudo a média do tempo de internação foi de 17 dias e os estudos que mais se aproximam de valores como esse são os de Einloft (2002)1 e com associação com doença neoplásica Lanetzki et al. (2012) ${ }^{15}$. 
Acredita-se que o tempo de internação na UTI pediátrica do HMCC foi mais elevado devido à ausência de uma unidade de cuidados semi-intensivos na instituição, o que incide em maior tempo de permanência de crianças com cuidados intermediários na unidade de terapia intensiva.

Os pacientes internados na UTI pediátrica são em sua maioria provenientes do próprio HMCC representando 76,6\% da amostra, este estudo está em concordância com Einloft $(2002)^{1}$ cuja análise apresentou predomínio de pacientes oriundos da própria instituição. Observou-se em muitos casos de internação/reinternação que as mesmas foram motivadas por causas sensíveis à atenção primária principalmente agravos respiratórios. Notam-se ainda poucos estudos sobre as internações por condições sensíveis à atenção primária, demonstrando a carência brasileira nesse setor que está intimamente ligado à eficácia da saúde pública15.

Durante a coleta de dados constatou-se casos de estenose subglótica que motivaram a reinternação de crianças que foram submetidas à intubação orotraqueal. A incidência desses casos vem aumentando levando em consideração fatores como tempo de intubação prolongado, diâmetro inadequado do tubo utilizado, múltiplas intubações, presença de balonete no tubo endotraqueal, maior duração de ventilação mecânica, menor idade e doenças pré-existentes na via aérea. Além disso, a ocorrência de pequenos traumas durante a passagem da cânula favorece o surgimento de estenoses $^{16}$.

O atendimento pela equipe multidisciplinar foi observado em $97,26 \%$ das internações na UTI pediátrica do HMCC nos anos 2016 e 2017, fato relevante principalmente por se tratar de um serviço pertencente ao SUS onde geralmente há escassez de recursos humanos, estabelece-se que a importância da atuação de qualquer membro da equipe é igual, isto é, toda atividade se reveste do caráter de indispensabilidade, apesar das diferentes ações junto ao paciente ${ }^{17}$.

Foram considerados atendimentos pela equipe multidisciplinar, os pacientes internados atendidos no mínimo uma vez durante a hospitalização na UTIP por pelo menos um dos integrantes dessa equipe, composta por: fisioterapeutas, terapeuta ocupacional, fonoaudióloga, psicóloga, assistente social e especialistas do corpo clínico (nefropediatria, neuropediatria, hematologia, nutrologia, pneumologia, cirurgia pediátrica, cirurgia plástica, oftalmologia, otorrinolaringologia, neurocirurgia, bucomaxilofacial, cirurgia cabeça e pescoço, cirurgia torácica, ortopedia, ginecologia, urologia, infectologia, dermatologia e psiquiatria). Do total de 329 internações no período de 2016/2017, 320 (97,26\%) tiveram atendimento pela equipe multidisciplinar, enquanto que apenas $9(2,74 \%)$ não foram atendidos por algum dos membros dessa equipe. Observou-se que poucos pacientes não foram atendidos pela equipe multidisciplinar, o que pode ser justificado por tempo de internação inferior a 2 dias e crianças que apresentaram internações em finais de semana ou período de férias desses profissionais, o que torna inviável a avaliação e atendimento dos mesmos.

Para as doenças respiratórias considerou-se período de maior incidência (sazonal) os meses de março a setembro, abrangendo estações mais frias como outono e inverno. Estudos mostraram um aumento de internações 
hospitalares por problemas respiratórios em meses do ano com temperaturas mais baixas e diminuição da precipitação típicos dos tempos secos de outono e inverno, corroborando com os resultados da presente pesquisa. Para as doenças neurológicas não se observou sazonalidade $\mathrm{e}^{18,19,20,21 .}$

Em um estudo sobre crianças com queimaduras ${ }^{22}$ obteve-se nos resultados prevalência maior de hospitalizações por esse motivo no verão com $41,81 \%$, bem como descrito na presente pesquisa a qual discorre que as queimaduras apresentam maior incidência nos meses de dezembro e janeiro. Ainda se levou em consideração os fatores de ordem socioeconômicos que podem ter relação com os casos de hospitalização por queimaduras. Casos cirúrgicos de apendicites tiveram aumento no último trimestre de cada ano analisado, fato que não foi encontrado em literaturas, e que merece ser investigado em pesquisas futuras.

Verificou-se que o mês que apresentou menor número de internações foi novembro de 2016 com 7 internações. Em contrapartida, março de 2017 apresentou o maior número de hospitalizações na UTIP totalizando 22 institucionalizações. A média de internações/mês nos anos de 2016 e 2017 foi de 7,0.

De modo geral observa-se uma prevalência maior de crianças internadas entre os meses que vão de março à setembro, os quais coincidem com as estações outono e inverno que apresentam características como: temperatura mais baixa e alterações da umidade relativa do ar predispondo ao acometimento por doenças respiratórias.

Pacientes acometidos por doenças neurológicas não foi observado sazonalidade, visto que as mesmas mantiveram uma distribuição linear durante os meses do ano. Em relação às crianças internadas com queimaduras, foram considerados sazonais para esse tipo de agravo os meses janeiro, julho e dezembro que coincidem com períodos de férias escolares. Fato este, que deixa as crianças mais vulneráveis à essa morbidade pelo fato de permanecerem mais em suas residências, muitas vezes sem a supervisão de um adulto.

Outro mês considerado sazonal foi junho devido às festividades juninas conhecidas culturalmente em nosso país. Para a sazonalidade em pacientes vítimas de queimaduras deve-se considerar também fatores socioeconômicos, já que algumas causas identificadas nessa afecção estavam relacionadas ao uso de meios alternativos para cocção dos alimentos quando o gás de cozinha não estava disponível por motivos de ordem econômica.

Para o grupo de doenças intitulado "outras" que engloba distúrbios hematológicos (como anemias), endocrinológicos (como a cetoacidose diabética), traumas e enfermidades cirúrgicas, não foram considerados como doenças sazonais por manterem uma regularidade de casos durante os meses dos anos 2016 e 2017. No estudo de Mota et al. (2002) 22 verifica-se o uso de ventilação mecânica em $25,30 \%$ dos pacientes, ou seja, em 84 desses e na presente pesquisa que tem um $\mathrm{N}$ próximo ao estudo comparado, 41,94\% ou 138 crianças foram submetidas à ventilação mecânica. Apesar desse número maior, a taxa de óbito dos pacientes que utilizaram essa terapêutica foi menor nesta 
análise, totalizando $10,33 \%$.

Quanto ao escore de risco de morte (PIM2) verificou-se que o mesmo apresentou um bom poder de discriminação e demonstrou uma boa acurácia assim como em um estudo semelhante em unidade de terapia intensiva pediátrica ${ }^{23}$. Em relação ao desfecho da internação neste estudo, 10,33\% evoluíram para óbito. Esse resultado se mostrou superior ao encontrado em alguns estudos como Lanetzki et al (2012) ${ }^{14}$ ao avaliar 433 prontuários revisados pelo sistema DATAMARTS e observou uma taxa de mortalidade de $1,8 \%$ e 0 estudo de

Alves et al (2014) $)^{24}$ ao avaliar 297 dados extraídos do "Livro de Alta, Admissão e Óbito" pertencente à UTIP internados de janeiro a dezembro de 2011, correspondendo a taxa de 7,7\% das mortalidades. Já o estudo de Benetti $(2015)^{25}$ evidenciou um número superior a esta pesquisa obtendo 14,3\% de mortalidade em uma UTI pediátrica de um hospital terciário do Rio Grande do Sul. Batista $(2015)^{12}$ em sua investigação de um hospital no Espírito Santo mostrou uma mortalidade também superior ao do presente estudo, com 15,6\%.

Recente estudo, revela que neonatos recém nascidos, apresentaram fatores de risco ligados ao sexo masculino, malformação congênita, asfixia perinatal, baixo peso ao nascer e prematuridade, como prevalentes para mortalidade neonatal no Brasil ${ }^{10}$. Neste estudo o sexo masculino e as doenças neurológicas (incluindo as malformações congênitas) mostraram-se de grande relevância nas crianças que foram a morte na UTI pediátrica. Os resultados gerais deste estudo mostram a importância de compreender, in locu hospitalar, os aspectos e características epidemiológicas de crianças internadas na UTI pediátrica de um hospital público, destacando-se, neste contexto, o poder que as mudanças estruturais, comportamentais e médicas para redução deste perfil.

\section{CONSIDERAÇÕES FINAIS}

As doenças neurológicas e de queimaduras foram consecutivamente superiores do que as doenças respiratórias nos anos de 2016 a 2017. Neste mesmo biênio a porcentagem das crianças que foram à óbito procedente do pronto socorro infantil e de serviços externos foi significantemente maior do que àquelas provenientes da enfermaria pediátrica e de outros setores hospitalares. 0 uso da ventilação mecânica foi maior nas crianças que evoluíram à óbito. $O$ valor do escore de risco de morte (PIM2) das crianças que foram à óbito foi relevante e superior ao das crianças que receberam alta. Estes resultados sugerem a importância de controle de fatores de risco para mortalidade durante o período assistencial das crianças hospitalizadas na UTIP, de forma a melhorar e aperfeiçoar a assistência clínica das doenças prevalentes nas crianças. 


\section{REFERÊNCIAS}

1. Einloft PR et al. Perfil epidemiológico de dezesseis anos de uma unidade de terapia intensiva pediátrica. Rev. Saúde Pública 2002;36(6):728-733.

2. Molina RCM, Marcon SS, Uchimura TT, Lopes EP. Caracterização das internações em uma unidade de terapia intensiva pediátrica, de um hospital-escola da região sul do Brasil. Ciência, Cuidado e Saúde, 2019;7:112-120.

3. Mendonça JG et al. Perfil das internações em Unidades de Terapia Intensiva Pediátrica do Sistema Único de Saúde no estado de Pernambuco, Brasil. Ciênc. Saúde Coletiva 2019;24(3):907-916.

4. Higuchi $\mathrm{CH}$ et al. Atenção Integrada às Doenças Prevalentes na Infância (AIDPI) na prática de enfermeiros egressos da USP. Rev. Gaúcha Enferm 2011;32(2):241-247.

5. Paixão AC, Barreto ES, Amaral JJF. Atenção Integrada às Doenças Prevalentes na Infância (AIDPI) e o ensino da pediatria na Universidade Federal de Sergipe. Rev Pediatr Ceará. 2003;4(1):51-4.

6. Granzotto JA et al. Fatores relacionados com a mortalidade neonatal em uma unidade de terapia intensiva neonatal na região sul do Brasil. Revista da AMRIGS, 2012;56(1):57-62.

7. Fundação Instituto Brasileiro de Geografia e Estatística. Projeção da População do Brasil. Dados de 2013, disponível em: https://brasilemsintese.ibge.gov.br/populacao/taxas-de-mortalidade-infantil.html, acessado em 25 de Março de 2018.

8. Geib LTC, et al. Determinantes sociais e biológicos da mortalidade infantil em coorte de base populacional em Passo Fundo, Rio Grande do Sul. Ciência \& Saúde Coletiva, 2010;15(2):363-370.

9. Cochran WG. Some methods for strengtering the commun X2 test. Biometrics 1954;10:417-451.

10. Siegel S, Castellan JR. Estatística não paramétrica para ciências do comportamento. $2^{\mathrm{a}}$ Ed. Artmed. Porto Alegre, 2006, 448p.

11. Veloso FC, Kassar LM, Oliveira MJ, Lima TH, Bueno NB, Gurgel RQ, et al. Analysis of neonatal mortality risk factors in Brazil: a systematic review and meta-analysis of observational studies. J Pediatr. 2019:95:519-30.

12. Batista NOW et al. Perfil clínico-epidemiológico de pacientes internados em unidade de cuidados intensivos pediátricos. J Human Growth Development. 2015;25(2):187-193.

13. Sena RR, Leite CR, Santana JJF, Vieira MA. Perfil das crianças atendidas na unidade de pediatria do Hospital Universitário Clemente de Faria, Montes Claros - MG. Unimontes Científica. 2006;8(1):118-28.

14. Lanetzki CS, Oliveira CAC, Bass LM, Abramovici S, Troster ED. O perfil epidemiológico do Centro de Terapia Intensiva Pediátrico do Hospital Israelita Albert Einstein. Einstein. 2012;10(1):16-21.

15. Morimoto T, Costa JSD. Internações por condições sensíveis à atenção primária, gastos com saúde e Estra- 
tégia Saúde da Família: uma análise de tendência. Ciência \& Saúde Coletiva 2017;22(3):891-900.

16. Machadol MC, Meloll ECM, Sál PM, Silval DA, et al. Estenose subglótica adquirida em recém-nascido sem fatores de risco tratados por laringoplastia: relato de caso. Residência Pediátrica. 2015;5(3):139-141.

17. Kamada C. Equipe multiprofissional em unidade de terapia intensiva. Rev. Bras. Enferm. 1978;31(1):60-67.

18. Murara PG, Mendonça M, Bonetti C. O clima e as doenças circulatórias e respiratórias em Florianópolis/SC. Rev Bras Geografia Médica e da Saúde, 2013;9(16):86-102.

19. Park JH, Chang YS, Ahn SY, Sung SI, Park WS. Predicting mortality in extremely low birth weight infants: comparison between gestational age, birth weight Apgar score, CRIB II score, initial

and lowest serum albumin levels. PLoS ONE. 2018;13:e0192232.

20. Demitto MO, Gravena AA, Dell'Agnolo CM, Antunes MB, Pelloso SM. High risk pregnancies and factors associated with neonatal death. Rev Esc Enferm USP. 2017;51:03208.

21. Miranda MH, Fernandes FE, Campos ME. Determinantes associados à mortalidade perinatal e fatores associados. Rev Enferm UFPE Online. 2017;11:1171-8.

22. Mota EM, Garcia PCR, Piva JP, Fritscher CC. A influência da desnutrição na utilização de ventilação mecânica em crianças admitidas em UTI pediátrica. J Pediatria, 2002;78(2):146-52.

23. Fraga JCS, Nogueira A, Palombini BC. Estenose subglótica em crianças. J Bras Otorrinolaringologia.1994;60(1):35-38.

24. Alves MVMFF, Bissiguini PO, Nitsche MJT, Olbrich SRLR, Luppi CHB, Toso LAR. Perfil dos pacientes internados em uma unidade de terapia intensiva pediátrica de um hospital escola do interior de São Paulo. Ciência Cuidado e Saúde 2014; 13(2):294-301.

25. Benetti MB. Caracterização das internações em uma unidade de terapia intensiva pediátrica - HUSM/RS. Rio Grande do Sul, 2015. Disponível em: https://repositorio.ufsm.br/handle/1/5847, acessado 2018 set 20. 